\section{RE: Evaluation of Small} Hepatocellular Carcinomas with Diffusion Weighted

\section{Imaging}

\author{
Ferhat Cuce, $M D^{1}$, Guner Sonmez, $M D^{2}$, \\ Emre Karaşahin, $M D^{3}$
}

${ }^{1}$ Department of Radiology, Van Military Hospital, Van 65020, Turkey; ${ }^{2}$ Department of Radiology, GATA Haydarpasa Training Hospital, Uskudar, Istanbul 34040, Turkey; ${ }^{3}$ Department of Obstetrics anda Gynecology, Van Military Hospital, Van 65020, Turkey

Index terms: Liver; Hepatocellular; Carcinoma; Diffusion

\section{Dear Sir,}

We are pleased to read the interesting article by Injoong Kim and colleagues (1) in the October 2012 issue of Korean Journal of Radiology. The authors examined the the histologic characteristics of hepatocellular carcinomas (HCCs) showing atypical enhancement patterns which would definitely add to the current literature. They found that HCCs showing atypical dynamic enhancement are usually smaller than typical HCCs. Since we have special interest on the diffusion magnetic resonance imaging (MRI) subject (2), we want to contribute information about the radiological evaluation of the small HCC nodules. HCC is the most common primary liver cancer and generally develops secondarily to chronic hepatitis or cirrhosis in elderly individuals. In the differential diagnosis of hepatocellular nodules up to $1 \mathrm{~cm}$ in diameter in the cirrhotic liver, regenerative nodules, low or high grade dysplastic nodules

Received October 20, 2012; accepted after revision December 31, 2012.

Corresponding author: Ferhat Cuce, MD, Department of Radiology, Van Military Hospital, Van 65020, Turkey.

- Tel: (90536) 8515475 - Fax: (90432) 2220244

- E-mail: ferhatcuce@hotmail.com

This is an Open Access article distributed under the terms of the Creative Commons Attribution Non-Commercial License (http://creativecommons.org/licenses/by-nc/3.0) which permits unrestricted non-commercial use, distribution, and reproduction in any medium, provided the original work is properly cited. and early or progressed HCC should be considered.

This poses a difficulty for the radiologist, especially in differentiation of dysplasia or HCC.

Diffusion weighted imaging (DWI) is virtually useless in detecting and characterizing borderline lesions in cirrhosis but ADC measurement of DWI may estimate the tumor grade of these HCCs since ADC tends to decrease in the order of well, moderately and poorly differentiated HCCs (3). Second, it was reported that if the liver function is normal, T2 weighted images may have the highest sensitivity among the other imaging parameters for differentiating HCCs 1 $\mathrm{cm}$ or smaller from benign hypervasculer lesions (4). Also, in this study (4) it was found that the combination of T2 sequence and DWI have high specificity in detection of HCC which is important - especially in elderly patients - against the risk of using contrast material. Third, due to the different histopathological features of liver parenchyme or HCC, DWI is useful in determining hepatic metastases commonly seen in elderly people and in atypical hemangioma cases which are defined by hypervasculer enhancement on the arterial phase (3). Lastly, after invasive treatments (transcatheter arterial chemoembolization, thermal ablation, partial hepatectomy and irradiation) of HCCs $1 \mathrm{~cm}$ or smaller, DWI helps to detect hypervascular intrahepatic HCC metastases or regional HCC recurrence by excluding the interference of early enhancing pseudolesions due to the postoperative arterioportal shunting (3).

\section{REFERENCES}

1. Kim I, Kim MJ. Histologic characteristics of hepatocellular carcinomas showing atypical enhancement patterns on 4-phase MDCT examination. Korean J Radiol 2012;13:586-593

2. Sonmez G, Cuce F, Mutlu H, Incedayi M, Ozturk E, Sildiroglu 0, et al. Value of diffusion-weighted MRI in the differentiation of benign and malign breast lesions. Wien Klin Wochenschr 2011;123:655-661

3. Kanematsu M, Goshima S, Watanabe H, Kondo H, Kawada $H$, Noda $Y$, et al. Diffusion/perfusion MR imaging of the liver: practice, challenges, and future. Magn Reson Med Sci 2012;11:151-161

4. Kim JE, Kim SH, Lee SJ, Rhim H. Hypervascular hepatocellular carcinoma $1 \mathrm{~cm}$ or smaller in patients with chronic liver disease: characterization with gadoxetic acid-enhanced MRI that includes diffusion-weighted imaging. AJR Am J Roentgenol 2011;196:W758-W765 